\title{
Predicting Incident Treatment-Resistant Depression: A Model Designed for Health Systems of Care
}

\author{
Joshua N. Liberman, PhD; Tigwa Davis, PhD; Jacqui Pesa, MSEd, PhD, MPH; \\ Wing Chow, PharmD, MPH; John Verbanac, PhD; Sara Heverly-Fitt, MSEd, PhD; \\ and Charles Ruetsch, PhD
}

\begin{abstract}
BACKGROUND: Major depressive disorder (MDD) is a prevalent and debilitating condition. While numerous treatment options are available, low treatment response and high remission rates remain common, leading to the concept of treatment-resistant depression (TRD): a classification applied to patients who fail multiple courses of therapy. A patient with TRD can only be identified after repeated, and often prolonged, therapeutic efforts.
\end{abstract}

OBJECTIVE: To use data readily available to integrated delivery networks to identify characteristics predictive of TRD among patients initiating pharmacotherapy for MDD.

METHODS: Decision Resources Group Real-World Data, an integrated medical/pharmacy claims and electronic health record dataset, was used to conduct a retrospective, longitudinal cohort study of patients with MDD who initiated antidepressant treatment between July 1, 2014, and December 31, 2015. Individuals were followed for 24 months to determine treatment resistance. Eligible individuals had integrated claims and electronic health record data available, completed at least 1 course of therapy of adequate dose and duration to achieve response, and had 30 months of continuous benefits eligibility ( 6 months before and 24 months after treatment initiation). Stepwise logistic regression and demographic, health history, health care utilization, medication, provider, and related characteristics were used to predict onset of TRD.

RESULTS: 35,246 people met eligibility and 7,098 (20.1\%) met TRD criteria after an average of 402 days. Significant predictors of TRD included patient age, diagnosis of insomnia and hypertension, psychiatric office visits, nurse telephonic encounters, anticonvulsant medication use, suicidality, physician specialty associated with index prescription, total prescription drug claims, unique antidepressants attempted, and duration of untreated illness (the lag between diagnosis and index prescription). The final model achieved an area under the curve $(A U C)=0.83$. Structured patient-generated health data, specifically, the Patient Health Questionnaire-2 and the Patient Health Questionnaire-9 were only reported for 542 patients $(1.5 \%)$.

CONCLUSIONS: TRD transition occurs after a prolonged treatment period, suggesting clinical inertia. Using data routinely available to integrated delivery networks and accountable care organizations, it is feasible to identify patients likely to qualify as treatment resistant. Monitoring risk factors may allow health systems to identify patients at risk for TRD earlier, potentially improving outcomes. Early identification of this at-risk population can allow for targeted resources for earlier intervention, more aggressive follow-up, and alternative treatment options. Furthermore, this model can be used to estimate future demand for specialized care resources, such as those delivered by mood disorder clinics.

J Manag Care Spec Pharm. 2020;26(8):987-95

Copyright $\odot 2020$, Academy of Managed Care Pharmacy. All rights reserved.

\section{What is already known about this subject}

Despite an array of treatment options, many patients with major depressive disorder (MDD) fail to respond, leading to a classification known as treatment-resistant depression (TRD).

TRD commonly affects more than 1 in 5 patients who have been diagnosed with MDD

Numerous demographic, clinical, treatment, and health factors have been identified as putting patients at increased risk of being defined as treatment resistant.

\section{What this study adds}

This study presents a functional predictive algorithm that can aid in the early identification of patients initiating antidepressant therapy who are at risk for TRD.

This study provides guidance on specific individual factors with the highest predictive accuracy: antidepressants attempted, total prescription claims, psychiatric office visits, nurse encounters, and suicidality.

Patients with TRD often seek care for a year or more before TRD is identified, suggesting substantial clinical inertia.

A ffecting nearly $6.6 \%$ of the U.S. population, major depressive disorder (MDD) is a common and debilitating condition. ${ }^{1-3}$ Those affected exhibit increased use of health care services, reduced productivity, and, in extreme cases, the inability to work. ${ }^{4}$ While numerous treatment options are available for MDD, including several classes of pharmaceutical therapy (e.g., selective serotonin reuptake inhibitors, serotonin-norepinephrine reuptake inhibitors, tricyclic agents, and serotonin modulators), ${ }^{5}$ alone or in combination with verbal psychotherapy such as cognitive behavioral therapy, and other verbal and procedural interventions, low response and high remission rates are still common. ${ }^{6-8}$

Because of the high treatment nonresponse rate in MDD, the concept of treatment-resistant depression (TRD) has emerged. ${ }^{9}$ TRD is commonly defined as failure to respond to at least 2 treatment regimens of adequate dose and duration. TRD is an important construct because individuals with TRD have greater disease burden, higher health care costs, and more affected employment than those who respond to treatment. ${ }^{10,11}$ 
By definition, a patient with TRD can only be identified after repeated, and often prolonged, therapeutic attempts. Health care providers, accountable care organizations, and insurers would benefit from the ability to identify TRD patients earlier in their course of therapy. Previous research to identify risk factors has been limited by small sample sizes in clinical populations, ${ }^{12-14}$ derive from clinical research studies, ${ }^{15,16}$ or rely on patient-reported or clinical data not routinely available in clinical practice or claims. ${ }^{17}$ One large scale study attempted to predict TRD using data only available at the point of initating therapy, which had low predictive accuracy. ${ }^{18}$

Despite varying populations, sample sizes, available data, and study designs, several characteristics are often cited as predictive of TRD. Among the most common characteristics are age; psychiatric comorbidities (i.e., anxiety, insomnia, suicidal risk, and select medical comorbidities); pain, diabetes, and thyroid disorder; depressive episode severity; treatment response; antidepressants used; and inpatient status. ${ }^{12-19}$ Data for many of these characteristics are available to health care organizations and are potentially useful to identify patients at risk for TRD.

The objectives of this study were to use data readily available to integrated delivery networks (i.e., electronic health record [EHR] and claims data) to characterize the population of patients with MDD, as well as those who meet criteria for TRD, and to use these characteristics to develop an algorithm that can be used by health systems to identify MDD patients with the greatest likelihood of manifesting TRD. Earlier identification of high-risk patients would provide the opportunity to (a) focus resources more efficiently, (b) monitor high-risk patients, and (c) provide interventions that may reduce delays in identifying and selecting effective treatments.

\section{Methods}

This is a retrospective, longitudinal cohort study that used commercially available integrated administrative claims and EHR data to identify and follow MDD patients who are initiating antidepressant pharmacotherapy.

\section{Sampling Frame and Data Source}

The sampling frame for this study was the population of patients with medical and pharmacy claims integrated with EHR data in Decision Resources Group's (DRG) Real-World Data (https://decisionresourcesgroup.com/solutions/realworld-data/). DRG's Real-World Data includes nationwide population samples of patient-specific medical and pharmacy claims and EHR data, inclusive of diagnoses, encounters, providers, pharmacy, vitals, orders, and results. The data source for this study was the subset of these populations with integrated claims and EHR data, which is estimated to be about 10 million individuals annually in the United States.

\section{Identification and Selection of Study Participants}

The study spanned a 4-year period from January 1, 2014, through December 31, 2017, and included 3 periods: casefinding (July 1, 2014-December 31, 2015); baseline (the 6-month period before the first antidepressant drug claim (index) in the case-finding period); and follow-up (the 24-month period after the index date). For inclusion in the study, a patient must have met all of the following criteria: (a) aged 18 years or older on the date of the initial antidepressant pharmacy claim; (b) $\geq 1$ antidepressant medication claim during the case-finding period, with at least 1 trial of antidepressant medication of adequate dose and duration as defined by the American Psychiatric Association (APA) ${ }^{10}$; (c) $\geq 6$-month period before index, with no claims for an antidepressant; (d) $\geq 2$ medical claims including a diagnosis of MDD (International Classification of Diseases, Ninth/ Tenth Revision, Clinical Modification [ICD-9-CM] codes 296.2, 296.3; ICD-10-CM codes F32.1,2,3,9; F33.1,2,3) within the 6-month baseline period through the 12-month period following index and continuous eligibility beginning 6 months before the index antidepressant medication claim and continuing through 24 months after index; and (e) did not have an ICD9/10-CM code indicating the presence of bipolar depression, dementia, psychosis or psychotic disorder, schizophrenia, Tourette Syndrome, or a dissociative or conversion disorder (Table 1).

\section{Treatment-Resistant Depression}

Each eligible MDD patient was assigned to 1 of 2 subgroups: TRD or non-TRD. Eligible participants met the TRD definition on the date they initiated a third antidepressant treatment course following 2 failed treatment courses of adequate dose and duration, as defined by the APA. ${ }^{5}$ The APA defines adequate dose for each drug individually, while adequate duration is defined as 4-8 weeks on therapy. For this study, we adopted the criteria for adequate duration, that is, 6 weeks on therapy and treatment failure, from Amos et al. (2018), ${ }^{10}$ with a treatment course considered as failed, if any of the following occurred: discontinuation ( $\geq 60$ days without medication); augmentation with an antipsychotic medication, lithium, transcranial magnetic stimulation, electroconvulsive therapy, vagus nerve stimulation, and stimulant medication; or switching to another antidepressant medication.

\section{Analysis}

Variable Construction. Variables were selected or derived using information from the administrative claims and EHR data files during the baseline period and follow-up periods. For TRD patients, the follow-up period was defined as the time between index and meeting criteria for TRD. Utilization and comorbidity statistics were derived from the 24-month period following the index. The follow-up period was imputed for non-TRD patients as the average time to TRD among 


\section{TABLE 1 Study Attrition}

\begin{tabular}{|c|c|c|c|}
\hline Step & Inclusion/Exclusion & Retained Cases & \% Retained \\
\hline 1. & $\begin{array}{l}\geq 1 \text { antidepressant medication fill (index fill, case-finding index date) and } \geq 1 \text { diagnosis of major } \\
\text { depressive disorder (ICD-9-CM: 296.2, 296.3; ICD-10-CM: F32.1,2,3,9; F33.1,2,3) during the } \\
\text { case-finding period (July 1, 2014-December 31, 2015) }\end{array}$ & 530,139 & 100.0 \\
\hline 2. & $\geq 6$-month antidepressant prescription naive period before case-finding index date & 312,983 & 59.0 \\
\hline 3. & Aged $\geq 18$ years at case-finding index date & 290,649 & 54.8 \\
\hline 4. & Absence of exclusionary diagnosis codes & 229,172 & 43.2 \\
\hline 5. & Continuous eligibility for 6 months before and 24 months after case-finding index date & 196,948 & 37.2 \\
\hline 6. & Integration of EHR and pharmacy claims data & 60,554 & 11.4 \\
\hline 7. & $\geq 1$ antidepressant medication dose on or after case-finding index date with adequate dose and duration & 60,058 & 11.3 \\
\hline 8. & $\begin{array}{l}\geq 2 \text { diagnosis of MDD (ICD-9-CM: 296.2, 296.3; ICD-10-CM: F32.1,2,3,9; F33.1,2,3) occurring during } \\
\text { the } 6 \text {-month naive period through the } 12 \text { months following the case-finding index date }\end{array}$ & 40,184 & 7.6 \\
\hline \multicolumn{2}{|r|}{ Total MDD population } & 35,246 & \\
\hline \multicolumn{4}{|c|}{ Assignment to TRD group } \\
\hline 9. & $\begin{array}{l}\text { Meets screening criteria for TRD: } 2 \text { failed antidepressant trials of adequate dose and duration with the } \\
\text { initiation of a third trial within the } 24 \text { months after case-finding index date }\end{array}$ & 7,098 & 20.1 \\
\hline 10. & Does not meet screening criteria for TRD & 28,148 & 79.9 \\
\hline
\end{tabular}

EHR = electronic health record; ICD-9/10-CM = International Classification of Diseases, Ninth/Tenth Revision, Clinical Modification; MDD =major depressive disorder;

$T R D=$ treatment-resistant depression.

TRD-designated patients. Unless otherwise noted, the presence of a code in either dataset was sufficient to qualify variables, which were developed for the following domains: patient demographics; medical and psychiatric comorbidities; health care utilization associated with psychiatric diagnosis or a medical diagnosis (claims and text from problem list); telephonic or nurse encounters (EHR data only); prescription drug use (claim counts for nonpsychiatric medications and claim counts and unique drug classes for psychiatric medications); suicidality; patient-generated health data (Patient Health Questionnaire-2 and Patient Health Questionnaire-9); and clinician affiliated with index antidepressant prescription. In addition, we calculated duration of untreated illness, defined as the time (in days) between the first occurrence of an MDD episode diagnosis code and the start of antidepressant therapy.

Statistical Analysis and Modeling. The exploratory analysis included bivariate comparisons of all variables by TRD status, with chi square, Student's t-test, and analysis of variance used as tests of significance. All patient, provider, and utilization statistics were calculated based on the entire analytical study sample. To develop and validate the predictive model, the analytical dataset was divided, 1:1 at random, into a dataset for developing (training) a predictive model and for validating the model and generating the model performance statistics. The randomization was tested by comparing the training and validation samples with regard to sex (\% female); age (mean, years); TRD status (\% of population); average time to TRD (days); comorbidity (Charlson Comorbidity Index); and race distributions. No significant differences were noted in any categories, except race (white: $55.5 \%$ vs. $54.8 \%$; black/African
American: $4.8 \%$ vs. $5.4 \%$; Asian: $0.3 \%$ vs $0.4 \%$; Other: $0.9 \%$ vs. $0.8 \%$; and unknown: $38.5 \%$ vs. $38.6 \% ; P<0.01$ ).

Stepwise (by domain) logistic regression analysis, applied to the training dataset, was used to develop an algorithm to predict "TRD" MDD status. Because of the number of available features, each "step" in the development of the regression model included variables within a domain (e.g., demographics and comorbidities). Variables with borderline significance $(P<0.10)$ were retained for the following steps. Variables not reaching statistical significance $(P<0.05)$ were dropped from the final model. The final regression model's performance was measured by applying the model to the validation dataset. All analyses were conducted using SAS Enterprise, version 9.4 (SAS Institute, Cary, NC).

\section{Results}

A total of 35,246 patients met eligibility. Overall, the MDD population was aged 56.4 years (SD 17.0 years); $71.5 \%$ were female; and $57.6 \%$ had commercial insurance, $21.8 \%$ Medicare, $14.7 \%$ Medicaid, and 5.9\% other insurance. After an average (SD) of 402 (106) days, 7,098 (20.1\%) patients met TRD criteria. Compared with non-TRD MDD patients, TRD patients were similar with regard to sex $(73.9 \%$ female vs. $71.0 \% ; P<0.001)$ and age (55.7 vs. 56.5 years; $P<0.001$ ); race and region of residence were not significant. The TRD population had significantly higher rates of most psychiatric and nonpsychiatric comorbidities measured (Table 2) and of nearly half the health care utilization measures (Table 3). Both groups had similar rates of documented telephone encounters (17.9\% vs. $17.2 \%$ ) and encounters with a nurse (1.3\% vs. $1.5 \%)$ in the EHR. $P$ values were not significant. 
TABLE 2 Comorbidities of the Non-TRD and TRD Patient Populations

\begin{tabular}{|c|c|c|c|c|c|}
\hline & \multicolumn{2}{|c|}{$\begin{array}{l}\text { Non-TRD } \\
\mathrm{n}=28,148\end{array}$} & \multicolumn{2}{|c|}{$\begin{array}{c}\text { TRD } \\
\mathbf{n}=7,098\end{array}$} & \multirow[b]{2}{*}{$P$ Value } \\
\hline & $\mathrm{n}$ & $\%$ & $\mathrm{n}$ & $\%$ & \\
\hline \multicolumn{6}{|l|}{ Gender } \\
\hline Female & 19,974 & 71.0 & 5,244 & 73.9 & $<0.001$ \\
\hline \multicolumn{6}{|l|}{ Age, years } \\
\hline $19-34$ & 3,666 & 13.0 & 738 & 10.4 & $<0.001$ \\
\hline $35-44$ & 3,808 & 13.5 & 1,060 & 14.9 & \\
\hline $45-54$ & 4,765 & 16.9 & 1,402 & 19.8 & \\
\hline $55-64$ & 6,392 & 22.7 & 1,829 & 25.8 & \\
\hline $65-74$ & 4,836 & 17.2 & 1,200 & 16.9 & \\
\hline $75+$ & 4,681 & 16.6 & 869 & 12.2 & \\
\hline \multicolumn{5}{|l|}{ Region of residence } & NS \\
\hline Northeast & 4,384 & 15.6 & 1,145 & 16.1 & \\
\hline Midwest & 8,427 & 29.9 & 2,108 & 29.7 & \\
\hline West & 2,395 & 8.5 & 566 & 8.0 & \\
\hline South & 12,926 & 45.9 & 3,279 & 46.2 & \\
\hline Unknown & 16 & 0.1 & 0 & 0.0 & \\
\hline \multicolumn{5}{|l|}{ Race } & NS \\
\hline White & 15,492 & 55.0 & 3,952 & 55.7 & \\
\hline Black & 1,457 & 5.2 & 333 & 4.7 & \\
\hline Asian & 103 & 0.4 & 22 & 0.3 & \\
\hline Other & 231 & 0.8 & 65 & 0.9 & \\
\hline Missing/declined/unknown & 10,865 & 38.6 & 2,726 & 38.4 & \\
\hline \multicolumn{5}{|l|}{ Insurance } & $<0.001$ \\
\hline Commercial & 16,241 & 57.7 & 4,053 & 57.1 & \\
\hline Medicaid & 4,002 & 14.2 & 1,165 & 16.4 & \\
\hline Medicare & 6,240 & 22.2 & 1,457 & 20.5 & \\
\hline VA/Other & 1,665 & 5.9 & 423 & 6.0 & \\
\hline \multicolumn{6}{|l|}{ Psychiatric } \\
\hline Anxiety & 10,519 & 37.4 & 3,031 & 42.7 & $<0.001$ \\
\hline Acute stress disorder & 85 & 0.3 & 28 & 0.4 & NS \\
\hline General anxiety disorder & 3,569 & 12.7 & 1,117 & 15.7 & $<0.001$ \\
\hline Panic attacks/panic disorder & 2,795 & 9.9 & 803 & 11.3 & $<0.001$ \\
\hline Social anxiety disorder & 107 & 0.4 & 37 & 0.5 & NS \\
\hline Post-traumatic stress disorder & 1,406 & 5.0 & 427 & 6.0 & $<0.001$ \\
\hline \multicolumn{6}{|l|}{ Sleepwake disorders } \\
\hline Sleep apnea & 2,721 & 9.7 & 792 & 11.2 & $<0.001$ \\
\hline Insomnia & 3,823 & 13.6 & 1,274 & 17.9 & $<0.001$ \\
\hline Hypersomnia & 143 & 0.5 & 64 & 0.9 & $<0.001$ \\
\hline \multicolumn{6}{|c|}{ Substance use and addiction disorders } \\
\hline Alcohol & 3,546 & 12.6 & 941 & 13.3 & NS \\
\hline Drugs of abuse & 2,303 & 8.2 & 599 & 8.4 & NS \\
\hline
\end{tabular}

A total of 4,185 (11.9\%) patients had evidence of suicidal ideation or attempts, recorded in either claims or EHR data. There was no difference in TRD rate among those patients with suicidal ideation only (9.9\% vs. 9.9\%). Compared with those patients with no documented suicidal ideation or attempt, for patients with a suicide attempt documented in the EHR, the TRD rate was elevated $(24.2 \%$ vs. $20.1 \%$; $P<0.05)$. Patientgenerated health data using the Patient Health Questionnaire-2 and the Patient Health Questionnaire-9 were identified in the Vitals and Results tables of the EHR data but were only reported for 542 patients (1.5\%).

Patients with TRD were more likely than non-TRD MDD patients to have a major depression diagnosis (MDD or major depressive episode) in the baseline period, before initiating index antidepressant therapy $(73.7 \%$ vs. $72.2 \%$; $P<0.05)$. TRD patients also had significantly greater medication use, filling on average 30.4 (vs. 16.7; $P<0.001$ ) prescriptions per year for psychiatric medications and 77.3 (vs. $50.4 ; P<0.001$ ) prescriptions 
TABLE 2 Comorbidities of the Non-TRD and TRD Patient Populations (continued)

\begin{tabular}{|c|c|c|c|c|c|}
\hline & \multicolumn{2}{|c|}{$\begin{array}{l}\text { Non-TRD } \\
\mathrm{n}=28,148 \\
\end{array}$} & \multicolumn{2}{|c|}{$\begin{array}{c}\text { TRD } \\
\mathbf{n}=7,098\end{array}$} & \multirow[b]{2}{*}{$P$ Value } \\
\hline & $\mathbf{n}$ & $\%$ & $\mathbf{n}$ & $\%$ & \\
\hline \multicolumn{6}{|l|}{ Medical } \\
\hline Cardiovascular conditions & 13,405 & 47.6 & 3,322 & 46.8 & NS \\
\hline Hypertension & 11,082 & 39.4 & 2,700 & 38.0 & $<0.05$ \\
\hline Hyperlipidemia & 6,939 & 24.7 & 1,796 & 25.3 & NS \\
\hline COPD & 5,835 & 20.7 & 1,587 & 22.4 & $<0.01$ \\
\hline Osteoarthritis & 5,645 & 20.1 & 1,531 & 21.6 & $<0.01$ \\
\hline Pain & 4,230 & 15.0 & 1,289 & 18.2 & $<0.001$ \\
\hline Fatigue & 4,152 & 14.8 & 1,192 & 16.8 & $<0.001$ \\
\hline Obesity & 3,561 & 12.7 & 1,072 & 15.1 & $<0.001$ \\
\hline Anemia & 2,758 & 9.8 & 772 & 10.9 & $<0.05$ \\
\hline Asthma & 2,422 & 8.6 & 692 & 9.7 & $<0.01$ \\
\hline Renal disease & 1,568 & 5.6 & 408 & 5.7 & NS \\
\hline Stroke/TIA & 1,561 & 5.5 & 426 & 6.0 & NS \\
\hline Diabetes & 1,543 & 5.5 & 346 & 4.9 & NS \\
\hline \multicolumn{6}{|l|}{ Suicidality } \\
\hline Ideation only & 2,798 & 9.9 & 701 & 9.9 & $<0.05$ \\
\hline Suicde attempt only & 147 & 0.5 & 52 & 0.7 & \\
\hline Suicidal ideation and attempt & 374 & 1.3 & 114 & 1.6 & \\
\hline Neither & 24,829 & 88.2 & 6,231 & 87.8 & \\
\hline
\end{tabular}

$C O P D=$ chronic obstructive pulmonary disease $; S=$ not significant $S D=$ standard deviation; $T I A=$ transient ischemic attack; TRD = treatment-resistant depression;

$V A=$ Veterans Affairs.

per year for nonpsychiatric medications. Patients with TRD had greater utilization of each psychiatric medication drug class: antidepressants (16.9 vs. 8.3; $P<0.001$ ), antipsychotics (2.2 vs. $1.5 ; P<0.001$ ), anxiolytics (5.3 vs. $3.2 ; P<0.001$ ), and anticonvulsants $(6.0$ vs. 3.8; $P<0.001)$. Use of nonpharmacologic interventions was less common, but patients with TRD were slightly more likely to attempt electroconvulsive therapy $(0.7 \%$ vs. $0.5 \% ; P<0.05)$ and transcranial magnetic stimulation ( $0.2 \%$ vs. $0.1 \% ; P<0.01)$

The index antidepressant medication was prescribed by several different types of providers. The most common prescriber was a primary care physician (55.9\%), followed by a psychiatrist (22.3\%), a physician assistant or nurse practitioner $(8.3 \%)$, and a pain specialist $(0.9 \%)$, with no differences in the distribution between TRD and non-TRD MDD cohorts.

The final model achieved an AUC $=0.83$. Predictors included age, insomnia, hypertension, psychiatric medications attempted, psychiatric office visits, nurse telephonic encounters, index antidepressant drug class, nonpsychiatric prescriptions (count), suicidality, and physician specialty (Table 4). Psychiatric office visits, nurse encounters, suicidal intent (ideation or attempt), and hypertension were associated with a lower odds of TRD. Treatment lag (in days) from initial MDD diagnosis to start of therapy, number of unique antidepressants attempted, claims for $\geq 1$ unique anticonvulsants, and total prescription claims were associated with a higher odds of TRD. Compared with initiating treatment with selective serotonin reuptake inhibitors, initiating with serotonin-norepinephrine reuptake inhibitors, serotonin modulators, or multiple drug classes simultaneously were all associated with lower odds of TRD.

\section{Discussion}

Algorithms applied to EHR and administrative claims data can allow providers, provider organizations, and payers to identify individuals at elevated risk of developing TRD and to intervene earlier in their course of treatment. Applied to individuals initiating therapy, early identification and delivery of supportive interventions may benefit patients with TRD who are exposed to a variety of treatment options throughout their course of therapy, often administered in a "trial and error" approach..$^{20}$

Overall, our predictive model achieved accuracy sufficient to implement in practice. However, the model requires an array of data inputs, as well as data elements, that are only measurable as the follow-up period and treatment efforts progress. Because elevated risk of TRD emerges with time, the optimal approach would be to apply the algorithm as a routine component of the care process among individuals initiating antidepressant therapy. This approach would be feasible for organizations with advanced computational capabilities and the ability to translate analytics into provider communications.

In the absence of those capabilities, our analysis still provides insights that can support organizations interested in 


\begin{tabular}{|c|c|c|c|c|c|}
\hline & \multicolumn{2}{|c|}{$\begin{array}{l}\text { Non-TRD } \\
n=28,148\end{array}$} & \multicolumn{2}{|c|}{$\begin{array}{c}\text { TRD } \\
\mathbf{n}=7,098\end{array}$} & \multirow[b]{2}{*}{$P$ Value ${ }^{a, b}$} \\
\hline & $\mathrm{n} /$ mean & $\% /(\mathrm{SD})$ & $\mathrm{n} / \mathrm{mean}$ & $\% /(\mathrm{SD})$ & \\
\hline \multicolumn{6}{|l|}{ Nurse encounters } \\
\hline No & 27,714 & $98.5 \%$ & 7,004 & $98.7 \%$ & NS \\
\hline Yes & 434 & $1.5 \%$ & 94 & $1.3 \%$ & \\
\hline \multicolumn{6}{|c|}{ Antidepressants, unique drugs } \\
\hline 1 & 15,928 & $56.6 \%$ & 992 & $14.0 \%$ & $<0.001$ \\
\hline 2 & 8,888 & $31.6 \%$ & 2,701 & $38.1 \%$ & \\
\hline 3 & 2,562 & $9.1 \%$ & 2,188 & $30.8 \%$ & \\
\hline 4 & 605 & $2.1 \%$ & 869 & $12.2 \%$ & \\
\hline $5+$ & 165 & $0.6 \%$ & 348 & $4.9 \%$ & \\
\hline \multicolumn{6}{|c|}{ Antipsychotics, unique drugs } \\
\hline 0 & 22,366 & $79.5 \%$ & 5,198 & $73.2 \%$ & \\
\hline 1 & 5,782 & $20.5 \%$ & 1,900 & $26.8 \%$ & \\
\hline \multicolumn{6}{|c|}{ Psychiatric service utilization ${ }^{c}$} \\
\hline Office visits & 4.6 & $(7.3)$ & 5.4 & $(8.8)$ & $<0.001$ \\
\hline Outpatient other & 0.7 & (3.6) & 0.8 & $(4.2)$ & NS \\
\hline ED visits & 0.04 & $(0.4)$ & 0.04 & $(0.3)$ & NS \\
\hline Outpatient hospital visits & 0.4 & $(1.8)$ & 0.5 & (1.9) & $<0.05$ \\
\hline Inpatient admissions & 0.5 & $(2.2)$ & 0.6 & $(3.5)$ & 0.01 \\
\hline \multicolumn{6}{|c|}{ Nonpsychiatric service utilization } \\
\hline Office visits & 16.8 & $(24.2)$ & 18.9 & $(26.1)$ & $<0.001$ \\
\hline Outpatient visits, other & 4.8 & $(12.8)$ & 5.2 & $(12.4)$ & $<0.05$ \\
\hline ED visits & 0.5 & $(2.3)$ & 0.5 & $(2.0)$ & NS \\
\hline Outpatient hospital visits & 3.7 & (8.6) & 4.3 & $(9.2)$ & $<0.001$ \\
\hline Inpatient admissions & 0.3 & $(1.1)$ & 0.3 & $(1.1)$ & NS \\
\hline \multicolumn{6}{|c|}{$\begin{array}{l}\text { aStatistical tests compare non-TRD group and TRD group. } \\
\text { bChi-square analysis was used to test categorical variables for significant differences, while Student t-test was used to test continuous variables for significant differences. } \\
\text { 'Services that include a psychiatric primary diagnosis. } \\
\text { ED=emergency department; TRD = treatment-resistant depression. }\end{array}$} \\
\hline
\end{tabular}

identifying at-risk individuals. Our study identified numerous predictive features, including patient demographics (age and gender), select comorbidities (insomnia and hypertension), health care resource utilization (psychiatric outpatient visits and telephonic encounters with nurses), suicidality, prescribing physician specialty, numerous measures of medication use (psychiatric and nonpsychiatric), and delayed initiation of therapy.

A principle challenge to comparing research results is the variable approaches to identifying and selecting patients; variability in the definition of TRD; and variability in the clinical, provider-generated, patient-generated, and administrative data used to identify predictors. Some studies of TRD predictors were limited to clinical populations with smaller sample sizes and clinical data that are not readily accessible in EHR or claims datasets. ${ }^{13,21}$ In a similarly constructed retrospective cohort analysis, limited to claims data only from nearly 231,000 commercially insured individuals initiating antidepressant treatment, Cepeda et al. (2018) reported on risk factors for TRD identified within the first year of treatment. ${ }^{18} \mathrm{At}$ a mean of 37 years, this population was substantially younger than ours, and the follow-up period was limited to 12 months, which may explain the substantially lower rate of TRD (10.3\%). Nonetheless, several risk factors were consistent, including indications that anxiety, insomnia, and pain were associated with patients who met criteria for TRD.

In our population, TRD was more likely among individuals who experienced a delay between the first major depressive diagnostic code and the initiation of treatment. These results are consistent with other research that indicates delayed treatment initiation results in poorer treatment response, ${ }^{21,22}$ poorer clinical outcomes and increased disability, ${ }^{23}$ and more severe long-term depression symptoms. ${ }^{24}$

Delays in initiating treatment are similar, conceptually, to the concept of clinical inertia as unwarranted variation in care. Using an empirical definition of treatment resistance that replicated that used by Amos et al., ${ }^{10}$ we observed an average time to TRD qualification of 402 days. Although there has been some recent criticism regarding the term "clinical inertia" for 
TABLE 4 Adjusted Odds Ratios and 95\% Cls, Final Model

\begin{tabular}{|c|c|c|c|c|c|}
\hline & Adjusted OR & $95 \% \mathrm{CI}$ & & Adjusted OR & $95 \% \mathrm{CI}$ \\
\hline \multicolumn{3}{|l|}{ Gender } & \multicolumn{3}{|l|}{ Prescriber, index medication } \\
\hline Female & 1.00 & - & Primary care & 1.00 & - \\
\hline Male & 0.91 & $(0.82-1.00)$ & Psychiatry & 0.91 & $(0.81-1.02)$ \\
\hline \multicolumn{3}{|l|}{ Age, years } & Pain specialist & 1.36 & $(0.89-2.07)$ \\
\hline $19-34$ & 1.00 & - & Specialist, other & 0.82 & $(0.71-0.96)$ \\
\hline $35-44$ & 1.20 & $(1.01-1.43)$ & \multirow{2}{*}{$\begin{array}{l}\text { Physician's assistant/nurse } \\
\text { practioner }\end{array}$} & \multirow{2}{*}{1.03} & \multirow{2}{*}{$(0.88-1.21)$} \\
\hline $45-54$ & 1.28 & $(1.08-1.52)$ & & & \\
\hline $55-64$ & 1.35 & $(1.14-1.59)$ & Missing & 0.92 & $(0.73-1.17)$ \\
\hline $65-74$ & 1.21 & $(1.01-1.44)$ & \multicolumn{3}{|l|}{ Index AD class } \\
\hline $75+$ & 0.97 & $(0.80-1.17)$ & SSRI & 1.00 & - \\
\hline \multicolumn{3}{|l|}{ Race } & Multiple index AD classes & 0.60 & $(0.53-0.67)$ \\
\hline White & 1.00 & - & SNRI & 0.74 & $(0.65-0.86)$ \\
\hline Black & 0.90 & $(0.74-1.11)$ & Serotonin modulator & 0.62 & $(0.51-0.74)$ \\
\hline Asian & 1.16 & $(0.54-2.47)$ & MAOI & 1.91 & $(0.24-15.1)$ \\
\hline Other & 1.22 & $(0.79-1.90)$ & Tetracyclics & 0.71 & $(0.53-0.95)$ \\
\hline Missing/declined & 0.94 & $(0.86-1.02)$ & Tricyclics & 0.86 & $(0.70-1.06)$ \\
\hline \multicolumn{3}{|c|}{ Comorbidities (presence of) } & Other & 1.06 & $(0.89-1.26)$ \\
\hline Insomnia & 1.12 & $(1.00-1.25)$ & \multicolumn{3}{|l|}{ Total prescription claims } \\
\hline Hypertension & 0.82 & $(0.75-0.91)$ & $1-24$ & 1.00 & - \\
\hline$\frac{\text { Office visits, psych }}{0}$ & 1.00 & - & $25-53$ & 2.36 & $(2.02-2.75)$ \\
\hline 1 & 0.55 & $(0.47-0.64)$ & $54-102$ & 3.12 & $(2.68-3.64)$ \\
\hline 2 & 0.54 & $(0.45-0.63)$ & $103+$ & 4.38 & $(3.73-5.13)$ \\
\hline $3+$ & 0.54 & $(0.46-0.64)$ & \multicolumn{3}{|l|}{ Unique ADs attempted } \\
\hline \multicolumn{3}{|l|}{ Nurse encounters } & 1 & 1.00 & - \\
\hline No & 1.00 & - & 2 & 5.0 & $(4.5-5.7)$ \\
\hline Yes & 0.57 & $(0.38-0.84)$ & 3 & 14.0 & $(12.3-16.0)$ \\
\hline \multicolumn{3}{|c|}{ Total anticonvulsants } & 4 & 23.4 & $(19.4-28.2)$ \\
\hline 0 & 1.00 & - & $5+$ & 31.1 & $(23.1-41.8)$ \\
\hline 1 & 1.20 & $(1.09-1.33)$ & \multicolumn{3}{|c|}{ Duration of untreated illness (days) } \\
\hline $2+$ & 0.91 & $(0.80-1.05)$ & 0 & 1.00 & - \\
\hline \multicolumn{3}{|l|}{ Suicidality } & $1-30$ & 1.05 & $(0.93-1.18)$ \\
\hline No presence & 1.00 & - & $31-60$ & 1.15 & $(1.00-1.32)$ \\
\hline Presence & 0.79 & $(0.69-0.90)$ & $61+$ & 1.17 & $(1.04-1.31)$ \\
\hline
\end{tabular}

being insufficiently specific to differentiate intentional delays in care from true clinical inaction, ${ }^{25}$ the literature is clear that both are present in the management of patients with chronic diseases. Aujoulat et al. (2014) propose a series of contributors to inertia, including provider knowledge of and attitude toward evidence-based guidelines; provider clinical judgment and experience in a particular clinical situation; provider awareness of patient attitudes, behaviors, and preferences; and providers' ability to make the appropriate decision. ${ }^{26}$

Treatment resistance may have a clinical definition at the individual level, but at the population level, TRD is a nonspecific classification that generally refers to patients who simply have not responded to the therapeutic options attempted rather than a clinical trait representing a truly intractable form of MDD. ${ }^{27}$ In our study, evidence that a classification as treatment resistant is, at least in part, tied to variation in management is found in the factors predictive of TRD, most importantly, that patients receiving outpatient psychotherapeutic care or under the care of a psychiatrist is associated with lower odds of TRD. These results suggest that more aggressive, guideline-aligned care can result in more patients with adequate treatment responses and fewer patients meeting criteria for TRD.

Another contributor to clinical inertia and the long delay to the assignment of TRD is likely the limited use of measurementbased care. Defined as "enhanced precision and consistency in disease assessment, tracking, and treatment to achieve optimal outcomes," ${ }^{28}$ measurement-based care incorporates the systematic administration and use of symptom rating scales to support clinical decision making, patient engagement, and monitoring of treatment response. A recently published 
randomized controlled trial reported that patients with major depression who received measurement-based care had substantially (and significantly) higher response and remission rates, both of which were achieved over a substantially shorter time period. ${ }^{29}$ These results are leading to calls for greater inclusion of measurement in clinical practice, particularly in behavioral health. ${ }^{30}$ In our dataset, the 9-item Patient Health Questionnaire, ${ }^{31}$ which is among the most common standard instruments used by clinicians to manage MDD, ${ }^{32}$ was reported for only $1.5 \%$ MDD patients.

\section{Strengths and Limitations}

Our research is consistent with previous research results and extends that work by identifying a comprehensive array of variables readily available to health systems, payers, and accountable care organizations. We analyzed a large-scale, national U.S. population of individuals initiating antidepressant treatment, providing an opportunity to control for numerous characteristics that have biased studies in smaller samples. The analytic design of this study was cross-sectional, meaning that some of the predictors derived from the same measurement period that also contained the data of conversion to TRD. As TRD may, in fact, be a physiologic trait that is revealed through multiple treatment failures, it can be argued that all predictors are cross-sectional. The set of predictive features can be used individually or in a full model, providing organizations with an opportunity to identify at-risk patients early in their treatment course.

The integrated clinical and claims dataset used were still restricted to transactional data with limited insights from the clinical narrative. Despite including EHR data, there was a near complete lack of patient-generated health data, which is essential for accurate assessment of depression severity and treatment response. Despite this, we were able to develop a predictive model with sufficient accuracy to have value in everyday practice. This suggests that the additional insights available within the clinical narrative could only enhance this model. Future research should focus on validation of the model in additional populations, enhancement of the model with insights from the narrative, and evaluation of efforts to implement the model.

\section{Conclusions}

Using data routinely available to integrated delivery networks and accountable care organizations, it is feasible to identify patients likely to qualify as treatment resistant. Early identification of this at-risk population can allow for targeted resources for earlier intervention, more aggressive follow-up, and alternative treatment options. Furthermore, this model can be used to estimate future demand for specialized care resources, such as those delivered by mood disorder clinics. Patient-generated health data, which is a consistent predictor of treatment response in smaller, clinical MDD populations, could prove an asset if routinely collected and used in measurement-based clinical care and may dramatically reduce the time to identify individuals as treatment resistant.

\section{Authors}

JOSHUA N. LIBERMAN, PhD; TIGWA DAVIS, PhD; SARA HEVERLY-FITT, MSEd, PhD; and CHARLES RUETSCH, PhD, Health Analytics, Columbia, Maryland. JACQUI PESA, MSEd, PhD, MPH; WING CHOW, PharmD, MPH; and JOHN VERBANAC, PhD, Janssen Scientific Affairs, Titusville, New Jersey.

AUTHOR CORRESPONDENCE: Joshua N. Liberman, PhD, Health Analytics, 9200 Rumsey Rd., Ste. 215, Columbia, MD 21045 Tel.: 410.997.3314; Email: j.liberman@healthanalytics.com.

\section{DISCLOSURES}

This project was sponsored by Janssen Scientific Affairs. Pesa, Chow, and Verbanac are employed by Janssen Scientific Affairs and report stock ownership in Johnson \& Johnson. Liberman, Davis, Heverly-Fitt, and Ruetsch are employed by Health Analytics, which received funding from Janssen Scientific Affairs for work on this project.

This study was presented as a poster at the U.S. Psych Congress; October 3-6, 2019; San Diego, CA

\section{ACKNOWLEDGMENTS}

The authors thank Antuane Allen who assisted with the statistical analysis.

\section{REFERENCES}

1. National Institute of Mental Health. Major depression. Available at: https://www.nimh.nih.gov/health/statistics/major-depression.shtml. Accessed June 12, 2020.

2. Kessler RC, Chiu WT, Demler O, Merikangas KR, Walters EE. Prevalence, severity, and comorbidity of 12-month DSM-IV disorders in the National Comorbidity Survey Replication. Arch Gen Psychiatry. 2005;62(6):617-27.

3. Ferrari AJ, Charlson FJ, Norman RE, et al. Burden of depressive disorders by country, sex, age, and year: findings from the global burden of disease study 2010. PLoS Med. 2013;10(11):e1001547.

4. Stewart WF, Ricci JA, Chee E, Hahn SR, Morganstein D. Cost of lost productive work time among US workers with depression. JAMA. 2003;289(23): 3135-44

5. Gelenberg AJ, Freeman MP, Markowitz JC, et al. Practice Guideline for the Treatment of Patients with Major Depressive Disorder. 3d. ed. American Psychiatric Association; 2010.

6. Hiranyatheb T, Nakawiro D, Wongpakaran T, et al. The impact of residual symptoms on relapse and quality of life among Thai depressive patients. Neuropsychiatr Dis Treat. 2016;12:3175-81.

7. Nil R, Lutolf S, Seifritz E. Residual symptoms and functionality in depressed outpatients: a one-year observational study in Switzerland with escitalopram. J Affect Disord. 2016;197:245-50.

8. Pigott HE, Leventhal AM, Alter GS, Boren JJ. Efficacy and effectiveness of antidepressants: current status of research. Psychother Psychosom. 2010;79(5):267-79. 
9. Nemeroff C. Prevalence and management of treatment-resistant depression. J Clin Psychiatry. 2007;68(Suppl 8):17-25

10. Amos TB, Tandon N, Lefebvre P, et al. Direct and indirect cost burden and change of employment status in treatment-resistant depression: a matched-cohort study using a US commercial claims database. J Clin Psychiatry. 2018;79(2):17ml1725.

11. Crown WH, Finkelstein S, Berndt ER, et al. The impact of treatmentresistant depression on health care utilization and costs. J Clin Psychiatry. 2002;63(11):963-71.

12. Balestri M, Calati R, Souery D, et al. Socio-demographic and clinical predictors of treatment resistant depression: a prospective European multicenter study. J Affect Disord. 2016;189:224-32.

13. Kautzky A, Baldinger-Melich P, Kranz GS, et al. A new prediction model for evaluating treatment-resistant depression. J Clin Psychiatry. 2017:78(2):215-22

14. Kautzky A, Dold M, Bartova L, et al. Refining prediction in treatmentresistant depression: results of machine learning analyses in the TRD III sample. J Clin Psychiatry. 2018;79(1):16ml1385.

15. Nie Z, Vairavan S, Narayan VA, Ye, J, Li QS. Predictive modeling of treatment resistant depression using data from STAR*D and an independent clinical study. PLoS One. 2018;13(6):e0197268.

16. Perlis RH. A clinical risk stratification tool for predicting treatment resistance in major depressive disorder. Biol Psychiatry. 2013;74(1):7-14.

17. Souery D, Oswald P, Massat I, et al. Clinical factors associated with treatment resistance in major depressive disorder: results from a European multicenter study. J Clin Psychiatry. 2007;68(7):1062-70.

18. Cepeda MS, Reps J, Ryan P. Finding factors that predict treatmentresistant depression: results of a cohort study. Depress Anxiety. 2018;35(7):668-73

19. Kautzky A, Dold M, Bartova L, et al. Clinical factors predicting treatment resistant depression: affirmative results from the European multicenter study. Acta Psychiatr Scand. 2019;139(1):78-88.
20. Al-Harbi KS. Treatment-resistant depression: therapeutic trends, challenges, and future directions. Patient Prefer Adherence. 2012;6:369-88.

21. de Diego-Adelino J, Portella MJ, Puigdemont D, Perez-Egea R, Alvarez E, Perez V. A short duration of untreated illness (DUI) improves response outcomes in first-depressive episodes. J Affect Disord. 2010;120(1-3):221-25.

22. Hung CI, Yu NW, Liu CY, Wu KY, Yang CH. The impact of the duration of an untreated episode on improvement of depression and somatic symptoms. Neuropsychiatr Dis Treat. 2015;11:2245-52.

23. Ghio L, Gotelli S, Cervetti A, et al. Duration of untreated depression influences clinical outcomes and disability. J Affect Disord. 2015;175:224-28.

24. Hung CI, Liu CY, Yang CH. Untreated duration predicted the severity of depression at the two-year follow-up point. PLoS One. 2017;12(9):e0185119.

25. Aujoulat I, Jacquemin P, Hermans MP, et al. Clinical inertia in general practice, a matter of debate: a qualitative study with 114 general practitioners in Belgium. BMC Fam Pract. 2015;16:13.

26. Aujoulat I, Jacquemin P, Rietzschel E, et al. Factors associated with clinical inertia: an integrative review. Adv Med Educ Pract. 2014;5:141-47.

27. Trevino K, McClinotck S, McDonald F, Vora A, Husain M. Defining treatment-resistant depression: a comprehensive review of the literature. Ann Clin Psych. 2014;26(3):222-32.

28. Harding KJ, Rush AJ, Arbuckle M, Trivedi MH, Pincus HA. Measurement-based care in psychiatric practice: a policy framework for implementation. J Clin Psychiatry. 2011;72(8):1136-43.

29. Guo T, Xiang YT, Xiao L, et al. Measurement-based care versus standard care for major depression: a randomized controlled trial with blind raters. Am J Psychiatry. 2015;172(10):1004-13.

30. Rush AJ. Isn't it about time to employ measurement-based care in practice? Am J Psychiatry. 2015;172(10):934-36.

31. Kroenke K, Spitzer RL, Williams JB. The PHQ-9: validity of a brief depression severity measure. J Gen Intern Med. 2001;16(9):606-13.

32. Siu AL, U.S. Preventive Services Task Force, Bibbins-Domingo K, et al. Screening for depression in adults: US Preventive Services Task Force Recommendation Statement. JAMA. 2016;315(4):380-87. 